\title{
Safety and efficacy of PNL vs RIRS in the management of stones located in horseshoe kidneys: A critical comparative evaluation
}

\author{
Bilal Eryildirim ${ }^{1}$, Eyup Veli Kucuk ${ }^{2}$, Gokhan Atis ${ }^{3}$, Metin Ozturk ${ }^{4}$, Temucin Senkul ${ }^{5}$, Murat Tuncer ${ }^{1}$, \\ Ahmet Tahra ${ }^{2}$, Turgay Turan ${ }^{3}$, Orhan Koca ${ }^{4}$, Ferhat Ates ${ }^{5}$, Omer Yilmaz ${ }^{5}$, Cenk Gurbuz ${ }^{6}$, Kemal Sarica ${ }^{7}$ \\ ${ }^{1}$ Health Sciences University, Dr. Lütfi Kirdar Training and Research Hospital, Urology Clinic, Istanbul, Turkey; \\ ${ }^{2}$ Health Sciences University, Umraniye Training and Research Hospital, Urology Clinic, İstanbul, Turkey; \\ ${ }^{3}$ Istanbul Medeniyet University, Goztepe Training and Research Hospital, Urology Clinic, Istanbul, Turkey; \\ ${ }^{4}$ Health Sciences University, Haydarpasa Numune Training and Research Hospital, Urology Clinic, Istanbul, Turkey; \\ ${ }^{5}$ Health Sciences University, Sultan Abdulhamid Han Training and Research Hospital, Urology Clinic, Istanbul, Turkey; \\ ${ }^{6}$ Medistate Hospital, Urology Department, Istanbul, Turkey; \\ ${ }^{7}$ Kafkas University, Faculty of Medicine, Urology Department.
}

\begin{abstract}
Summary Aim: To assess the efficacy and safety of two different techniques (Percutaneous nephrolithotomy (PNL) vs Retrograde intrarenal surgery (RIRS)) in the management of stones in patients with horseshoe kidneys (HSK).

Patients and methods: Departmental files of 88 cases with radiopaque kidney stones in horseshoe kidneys undergoing two different approaches (PNL vs RIRS) were evaluated with respect to the success and complication rates of in a retrospective manner. In addition to the factors related with the procedures (success and complication rates, additional procedures), patient and stone characteristics were all well evaluated. Findings obtained in both groups were evaluated in a comparative manner with respect to the statistical significance. Results: Stone free rates were comparable in both groups after 1-week period (81.6\% PNL vs $80 \%$ RIRS). As well as 3 months evaluation (84.2\% PNL and $82.0 \%$ RIRS). The percentage of the cases with residual fragments $(>4 \mathrm{~mm}$ ) were similar in both groups and while all PNL procedures were completed in one session, mean number of RIRS sessions was higher (1.22 \pm $0.05)$. Mean duration of the procedure was slightly higher in RIRS group and based on Clavien scoring system, despite a higher risk of $\mathrm{Hb}$ drop noted in patients treated with PNL, all complication rates were found to be similar in both groups. Conclusion: Our results demonstrate that of the available minimally invasive treatment alternatives, both PNL and RIRS could be safe and effective alternatives for renal stone removal in patients with HSK.
\end{abstract}

KEY WORDS: Horseshoe kidney; Percutaneous nephrolithotomy; Retrograde intrarenal surgery; Renal stone; Ureteroscopy.

Submitted 8 March 2018; Accepted 29 April 2018

\section{INTRODUCTION}

Being observed in approximately 1 in 400 to 1 in 666 births, horseshoe kidney (HSK) is the most common renal fusion anomaly $(1,2)$. Anterior displacement of the renal pelvis and high insertion of the involved ureter cause urinary abnormal drainage with flow hinderance and urinary stasis in the collecting system which may result in stone formation (1). The reported incidence of urolithiasis in patients with HSK varies between $20 \%$ to $60 \%$ in different series $(3,4)$.

On the other hand again, abnormal position of the kidney due to congenital fusion abnormality and unusual course of the ureter over the isthmus bring the management of stones in such kidneys into more challenging position. Although open surgery has been performed commonly in the past, currently all available minimal invasive treatment alternatives such as extracorporeal shock wave lithotripsy (SWL), retrograde intrarenal surgery (RIRS) and percutaneous nephrolithotripsy (PNL) are being performed with varying success rates (4).

Of these alternatives although SWL has been performed as a practical and well tolerated procedure by the majority of cases, success rates in such patients are highly variable and stone free rates (SFR) of 31-100\% were reported in the literature (5-8). Although adequate fragmentation can be achieved by SWL, the anatomic abnormalities may prevent fragment passage in a substantial number of these patients.

As a second alternative, PNL has already been found to be efficient in the management of relatively large stones cases with HSK with varying success rates. Due to the relatively lower position of the kidneys most upper as well as mid renal calyces are found to be located below the twelfth rib, thereby making the supracostal puncture relatively safe in these patients. However, despite the higher success rates obtained, the invasive nature of this method and higher risk of major complications (complication rates $14.3-29.2 \%$ ) led the endourologists to look for more feasible and less invasive options. Stone clearance rates after PNL in series with relatively limited number of renal units have been reported to be 75 $87.5 \%(3,9,10)$.

Last but not least, retrograde intrarenal surgery by using flexible ureterorenoscope has also been shown to be an effective management option; the success rate and associ- 
ated complications of this method have not been well documented in such patients. Despite acceptable stone free rates reported in some trials with relatively limited number of cases, due to the anatomical abnormality, a second look is usually required to render the patient completely stone free. A recent review article focusing on the management of renal stones in HSK demonstrated that RIRS may provide acceptable stone free rates (78\%) (11). In this present study we aimed to evaluate the clinical efficacy of two different approaches, RIRS combined with holmium laser lithotripter and PNL, in treating renal calculi in horseshoe kidney.

\section{Patients And methods}

The medical records of 88 cases with radiopaque renal stones in horseshoe kidneys treated with two different types of procedures (PNL vs RIRS) in 7 different centers between 2007 and 2016 were reviewed. Study protocol was approved by the Ethics Committee of the Hospital. All steps of the study were planned and applied carefully according to Helsinki Declaration.

Depending on the type of the procedure applied, cases with horseshoe kidneys were divided into two different groups; in Group $1(\mathrm{n}=38)$ cases were treated with PNL and in Group $2(n=50)$ cases were treated with RIRS. All treatment related parameters (stone free rates, number of sessions, treatment duration, hospitalization time, presence of the residual fragments, complications as well as the need for additional interventions) were noted and evaluated between two groups in a comparative manner. Preoperative evaluation

Prior to the above mentioned procedures, in addition to the preoperative laboratory tests including urinalysis, renal functional parameters, complete blood count and prothrombin concentration, urine culture and sensitivity test was performed in all patients with pyuria. Patients with urinary tract infection (UTI) were treated before the procedure with an appropriate antibiotic regimen. All patients were given prophylactic antibiotics at the time of anesthesia induction.

In addition to kidney-ureter-bladder (KUB) and urinary system ultrasonography (USG), a non-contrast abdominopelvic computed tomography (NCCT) were performed for radiologic evaluation. NCCT was also applied to assess the final stone free rates after 3-months and/or when there is any suspicion for residual fragments. Both procedures were performed under general anesthesia, a prone position was used for PNL and RIRS procedures were performed in lithotomy position. While the stone disintegration was performed with Ho-YAG laser during RIRS, all stones were fragmented by using pneumatic lithotriptor during PNL.

\section{Postoperative evaluation}

Success rates in all patients were evaluated one week and 3 months after

\section{Table 1.}

the last session of PNL and RIRS with KUB and/or urinary system USG to check the degree of stone fragmentation and the presence of obstruction (hydronephrosis) if present. The ultimate success rates were defined as no stone detectable or the presence of fragments $<4 \mathrm{~mm}$ on low dose non-contrast CT imaging for all patients.

Statistical Analysis: The Prism 5.0 (GraphPad Software, San Diego, CA) was used for the statistical analysis. Data are presented as mean standard error of mean. Student's $t$ test was used for both comparison of descriptive statistical methods and evaluation of quantitative data and chi-square test were used to compare the qualitative data between two groups; $\mathrm{p}<0.05$ was consider significant.

\section{Results}

Departmental files of 88 cases with kidney stones in horseshoe kidneys (56 male and 32 female (M/F: 1.75) undergoing two different approaches (PNL vs RIRS) were evaluated with respect to the success and complication rates of each procedure in a retrospective manner. While the age of the cases ranged from 19 to 60 years (mean 41.161.25); the overall mean size of the stones were $20.590 .75 \mathrm{~mm}(7-42 \mathrm{~mm})$. Patients and stone related factors are summarized in Table 1.

All PNL procedures were completed in one session, but the mean number the sessions for cases undergoing RIRS was $1.220 .05(p=0.0064)$. Although mean duration of the procedure was slightly longer in RIRS group $(p=0.9075)$, mean radiation exposure time was higher in PNL group when compared cases undergoing RIRS ( $\mathrm{p}<0.0001)$. On the other hand, while the mean duration of hospital stay was 3.150.24 days (1-10) in patients undergoing PNL procedure; this value was 1.580 .20 days ( 1 to 7 ) in RIRS group ( $p<0.0001$ ) (Table 2). While a nephrostomy tube (14 F) has been placed in 36 cases $(94.7 \%)$ after the PNL procedure, a double J stent was inserted in 42 of 50 cases undergoing RIRS procedure $(84.0 \%)$. Evaluation of the degree of hydronephrosis revealed a mean value of 2.050.14 dilatation in Group 1 and 1.460.16 in Group 2, there was a statistically significant difference on this aspect $(\mathrm{p}=0.0087)$.

Evaluation of patient and stone characteristics in both groups.

\begin{tabular}{|c|c|c|c|c|}
\hline & $\begin{array}{l}\text { Overall } \\
n=88\end{array}$ & $\begin{array}{c}\text { Group } 1 \text { (PNL) } \\
n=38\end{array}$ & $\begin{array}{c}\text { Group } 2 \text { (RIRS) } \\
n=50\end{array}$ & $\mathbf{P}^{*}$ \\
\hline Age (year) & 41.161 .25 & 42.970 .87 & 39.781 .68 & 0.1434 \\
\hline BMI $\left(\mathrm{kg} / \mathrm{m}^{2}\right)$ & 28.270 .42 & 29.150 .59 & 27.600 .56 & 0.0643 \\
\hline Stone size $(\mathrm{mm})$ & 20.590 .75 & 21.551 .14 & 19.460 .56 & 0.4638 \\
\hline HU (Hounsfield unit) & 97123.46 & 95734.29 & 98226.31 & 0.5496 \\
\hline Degree of hydronephrosis (grade) & 1.720 .11 & 2.050 .14 & 1.460 .16 & 0.0087 \\
\hline \multicolumn{5}{|l|}{ Stone location; n, (\%) } \\
\hline Renal pelvis & $30(34.1)$ & $12(31.6)$ & $18(36.0)$ & 0.8208 \\
\hline Upper calyx & $8(9.1)$ & $3(7.9)$ & $5(10.0)$ & 1.0000 \\
\hline Middle calyx & $9(10.2)$ & $3(7.9)$ & $6(12.0)$ & 0.7263 \\
\hline Lower calyx & $22(25.0)$ & $9(23.7)$ & $13(26.0)$ & 1.0000 \\
\hline Multiple calyces & $19(21.6)$ & $11(28.9)$ & $8(16.0)$ & 0.1923 \\
\hline
\end{tabular}


Table 2.

Evaluation of the outcomes of the procedures in terms of success rates as well as early post-operative follow-up data.

\begin{tabular}{|lcccc|}
\hline & $\begin{array}{c}\text { Overall } \\
\mathbf{n}=\mathbf{8 8}\end{array}$ & $\begin{array}{c}\text { Group } \mathbf{1} \text { (PNL) } \\
\mathbf{n}=\mathbf{3 8}\end{array}$ & $\begin{array}{c}\text { Group 2 (RIRS) } \\
\mathbf{n = 5 0}\end{array}$ & P* \\
\hline Mean duration of the procedure (min) & 77.342 .71 & 75.842 .64 & 80.265 .13 & 0.9075 \\
\hline Mean number of sessions & 1.110 .03 & 1.000 .0 & 1.220 .07 & 0.0064 \\
\hline $\begin{array}{l}\text { Stone free rate; } \mathbf{n},(\%) \\
\text { 1. Week }\end{array}$ & $71(80.7)$ & $31(81.6)$ & $40(80.0)$ & 0.7886 \\
3. Month & $73(82.9)$ & $32(84.2)$ & $41(82.0)$ & 0.7820 \\
\hline Residual stone $>\mathbf{4} \mathbf{~ m m} \mathbf{n},(\%)$ & $12(13.6)$ & $5(13.2)$ & $7(14.0)$ & 0.7559 \\
\hline Mean fluoroscopy time (sec.) & 30.805 .40 & 69.249 .43 & 20.621 .81 & $<0.0001$ \\
\hline Mean drop in hb levels (g/dL) & 0.740 .10 & 1.550 .15 & 0.130 .07 & $<0.0001$ \\
\hline Mean hospital stay (day) & 2.460 .12 & 3.150 .24 & 1.580 .20 & $<0.0001$ \\
\hline Auxiliary procedures; $\mathbf{n},(\%)$ & $6(6.8)$ & $3(7.9)$ & $3(6.0)$ & 1.0000 \\
\hline *Comparison between Group 1 and Group 2. & & & & \\
\hline
\end{tabular}

Table 3.

Evaluation of the type and grade of complications according to modified Clavien classification in both groups.as early post-operative follow-up data.

\begin{tabular}{|c|c|c|c|c|c|}
\hline \multicolumn{2}{|c|}{ Gradecomplication } & \multirow{2}{*}{$\begin{array}{c}\begin{array}{c}\text { Overall } \\
\mathbf{n = 8 8}\end{array} \\
14(15.9)\end{array}$} & \multirow{2}{*}{$\begin{array}{c}\text { Group } \mathbf{1} \text { (PNL) } \\
\mathbf{n = 3 8} \\
6(15.8)\end{array}$} & \multirow{2}{*}{$\begin{array}{c}\text { Group } 2 \text { (RIRS) } \\
\mathbf{n}=\mathbf{5 0}\end{array}$} & \multirow{2}{*}{$\begin{array}{c}\text { P* } \\
0.2274\end{array}$} \\
\hline 1 & Fever > 38 0c; n, (\%) & & & & \\
\hline & $\begin{array}{l}\text { Hemorrhage/hematuria not } \\
\text { requiring blood transfusion; n, (\%) }\end{array}$ & $8(9.0)$ & $5(1.8)$ & $3(3.6)$ & \\
\hline 2 & $\begin{array}{l}\text { Hemorrhage/hematuria requiring } \\
\text { blood transfusion; } n,(\%)\end{array}$ & $4(4.5)$ & $3(7.9)$ & $1(2.0)$ & 0.3113 \\
\hline$\overline{3 a}$ & $\begin{array}{l}\text { Double J stent placement for } \\
\text { ureteral stone; } n,(\%)\end{array}$ & $3(3.4)$ & $2(5.2)$ & $1(2.0)$ & 0.4916 \\
\hline$\overline{3 b}$ & $\begin{array}{l}\text { Endoscopic treatment for } \\
\text { ureteral stone; } n,(\%)\end{array}$ & $6(6.8)$ & $3(7.9)$ & $3(6.0)$ & \\
\hline
\end{tabular}

the larger residual fragments ( $>4 \mathrm{~mm}$ ) in 7 cases of RIRS group, although 1 case did pass the fragment spontaneously, fragments passed into the ureter in 3 cases and were removed again with URS during 3-months follow-up period. A double J stent was again inserted in only 1 of these 3 cases prior to URS due to the obstruction induced by ureteral stones.

PNL was unsucessful in 2 cases due to the difficulty in accessing the stones (5.3\%) and RIRS was performed in these cases. On the other hand, RIRS was unsuccessful in 3 cases $(6.0 \%)$ because of the difficulty in accessing to the lower pole. A mini-PNL was performed in all these 3 cases with successful stone removal. All these cases were excluded from the study program and were not included in any of these groups (Table 2).

\section{Evaluation of complication rates}

All complications observed in both groups were classified by using modified Clavien scoring system (12).

Based on this classification, while 11 cases (28.9\%) in PNL group demonstrated grade 1 complications, this number was again 11 (22.0\%) in RIRS group ( $p=0.2274)$. Regarding these complications, although fever after the procedures were noted in a total of 14 cases in both groups (6 in PNL and 8 in RIRS group) none of these cases demonstrated sepsis during follow-up.

Evaluation of the results obtained with two different techniques revealed following findings.

\section{Evaluation of the success as well as auxiliary procedure rates}

The overall percentage of the cases with no stones detectable or demonstrating fragments $<4 \mathrm{~mm}$ after one week period was $80.7 \%$ and the stone free rates were comparable in both groups after 1-week period (81.6\% for PNL group and $80.0 \%$ for RIRS group, $\mathrm{p}=0.7886$ ). This was also true during post-operative 3 months evaluation period where $84.2 \%$ of cases treated with PNL were stone free when compared with the cases undergoing RIRS procedure $(82.0 \%)(\mathrm{p}=0.7820)$.

Evaluation of the residual fragments (RF) sizing $>4 \mathrm{~mm}$ demonstrated that 5 cases (13.2\%) in PNL group; 7 cases in RIRS group (14.0\%) had such larger residual fragments during early (1-week) follow-up evaluation. During the 3 -months follow-up period while 1 case in PNL group passed these fragments spontaneously, fragments passed into the ureter in the other 3 cases. A JJ stent have already been inserted in 2 of these 3 cases due to the severe obstruction. Fragments were removed with URS in these 3 cases. 1 case was asmptomatic with RF requiring no intervention. On the other hand, regarding
Morover, regarding the grade 3 complications while 5 cases in PNL group did show such complications, 4 cases in RIRS group had this kind of complications $(p=0.4916)$ (Table 3$)$. Although evaluation of the rate of hemoglobin drop in both groups showed a statistically significant difference between two groups in ( $p<$ 0.0001 ); 3 cases in PNL group and 1 case in RIRS group required blood transfusion ( $\mathrm{p}=0.3113$ ) without any significant difference between two groups. No severe (Grade IV-V) complication was noted in any case. Last but not least stone street formation due to the fragment passage into the ureter has been observed in 1 case undergoing PNL in whom a double J stent placement and ureteroscopic stone removal have been performed.

\section{Discussion}

Representing the most common congenital renal fusion anomaly; HSK is mainly associated with the abnormal position of the kidney associated with the unusual course of upper ureter over the isthmus (2). Published data and clinical experience have clearly shown that these anatomical abnormalities cause impaired renal pelvic drainage due to ureteropelvic junction obstruction and resultant hydronephrosis may cause not only with 
stone formation but also makes the stone removal procedures more challenging.

Concerning the stone management principles in these patients, all available minimally invasive stone removal procedures namely SWL, PNL and RIRS have been applied as with varying stone-free rates in these patients. Although limited, published data so far has demonstrated that while altered pyelocalyceal system anatomy and high ureteropelvic junction position may lead to relatively poor results of SWL and RIRS; similar to the kidneys with normal anatomy, PNL has been reported as an efficient treatment modality for stones located in HSK $(13,14)$.

Related with this issue, as a non-invasive and safe alternative, although SWL has been well tolerated by the majority of patients, stone-free rates have been reported to be significantly lower after this procedure. When compared with the patients demonstrating normal kidneys, lower efficiency of this approach could be well explained with some certain factors such as greater skin-to-stone distance (particularly for calyceal stones) value and of more importantly restricted urinary drainage. However, despite the lower stone free rates reported to be changing between $31-100 \%$ (5-8), higher risk of complications associated with PNL performed for relatively large stones in HSK (14.3-29.2\%) make SWL also RIRS more feasible and safe options in selected cases $(3,9,10)$.

On the other hand again, developments in instruments technology and increasing experience in minimally invasive management of stones have changed the treatment concepts of urinary calculi meaningfully over the past two decades. As a result, PNL has become the standard of care for the treatment of large $(>2 \mathrm{~cm})$ stones with its evidently higher stone free rates in a single session (15, 16). However, it is well-known that these higher stonefree rates are being obtained at the expense of some certain severe complications (16).

In the light of the evident lower stone free rates after SWL due to the certain factors mentioned above however, accumulated data so far clearly demonstrate that PNL may constitute a preferred alternative in cases with HSK in experienced hands $(3,17)$.

Related with this issue, clinical experience showed that while access to the upper pole calyces during PNL often requires a supracostal approach with the associated risk of pleural injury in patients with normal renal anatomy, due to the relatively lower level of the upper pole, a supracostal puncture appears to be relatively safe in these kidneys. Access through the lower pole are not usually recommended due to the posterior location of these calyces. Furthermore, upper-pole access in HSK allows the surgeon to remove all stone burden in one session, as the alignment of the nephroscope with the long axis of the kidney aids manipulation of the scope into the upper calyces, renal pelvis, lower calyces, UPJ (ureteropelvic junction) and proximal ureter in a practical and safe manner. However, as mentined above despite the higher success rates obtained, the complicated nature of this method and higher risk of major complications led the endourologists to look for more feasible and less invasive options $(3,9,10)$.

Evaluation of the literature with respect to the success as well as complications rates of PNL reported in HSK demonstrated varying rates in studies with limited number of cases. In a single session operation using only one tract without any flexible nephroscope, Etemadian M. et al. have reported a stone free rate of $71.4 \%$ with a transfusion rate of $4.76 \%$ being encountered only in 1 patient (18). In their original study again, El Ghoneimy MN et al. treated 21 HSK renal units bearing a single pelvic stone in $52 \%$ of the cases and a staghorn calculi in $14 \%$ of the cases and $85.7 \%$ of the cases were stone free after the procedure with negligible complications (19). In a study of 24 cases with HSK again, Raj et al. performed upperpole access in the majority of the cases $(63 \%)$ and reported only one pneumothorax formation. They were also able to note that the use of flexible nephroscope was usually required for a satisfactory stone clearance rate, given as $87.5 \%$ (3). Last but not least, in relatively larger series of cases Symons SJ et al. treated stones in 47 cases with HSK and demonstrated that the number of PNL sessions required for stone free status depended on both the stone configuration and the size as well. PNL was used in 60 renal units of 47 patients with a clearance rate of $77 \%$ at one session and while 12 (71\%) of the 17 patients demonstrating multiple stones were stone-free in one session; 30 (86\%) out of 35 with neither multiple nor staghorn stones were completely stone free (10).

An alternative to the percutaneous approaches in these cases is flexible ureteroscopy, also referred to as RIRS that has been basically used to treat the lower pole stones resistant to SWL (20). As a result of increasing experience, relatively larger renal stones have also been treated with this approach and first serie of RIRS in HSK patients was published in 2005 where a stone clearance was achieved in 3 of the 4 patients (21). Following this publication, in their original study Molimard et al. treated 17 patients with HSK and 15 patients (88.2\%) with mean stone size of $16 \mathrm{~mm}$ were stone free in one session.

The success rate was comparable to PNL and better than SWL studies with no major complications and 7 (41.2\%) patients required more than one session of RIRS (22).

In another study, 25 renal stones in 20 patients were treated with RIRS and a SFR of $70 \%$ was reported. The authors found the success rates comparable with PCNL and better than SWL with the advantage of lower complication rates (23).

Currently RIRS and holmium - YAG laser lithotripsy is being increasingly used in the treatment of stone disease particularly in HSK patients. SFR of RIRS in the management of HSK patients were reported to be $70 \%$ and 88.2 $\%$ in the two recently published studies $(22,23)$. As mentioned above altered orientation of the calyces and high insertion of the ureter coupled with the increased likelihood of UPJ obstruction, endourologits may face evident technical difficulties during RIRS procedure in these cases.

In a relatively higher number of cases with HSK patients (32 stones in 23 patients) undergoing RIRS, Gokce IM et al. reported a SFR of $73.9 \%$ with acceptable and comparable complication rates ( 4 of the 23 patients) with previously published series (24).

With the advancement of technology, smaller activelydeflectable flexible ureteroscopy (f-URS), equipped with 
holmium laser lithotripter and nitinol baskets or graspers increased the effectiveness of RIRS in terms of stone-free rates.

In this present study we mainly aimed to assess the efficacy and safety of two different techniques (PNL vs RIRS) in the management of renal stones in patients with horseshoe kidneys. To our knowledge our study is the first one comparing the efficacy of two different valuable alternatives in the management of renal stones in HSK cases.

Evaluation of our results clearly showed that SFR's were comparable in both groups during 1-week $(81.6 \%$ vs $80.0 \%)$ as well as post-operative 3 months evaluation period. The rate of larger residual fragments $(>4 \mathrm{~mm})$ were again similar in both groups. However, while all PNL procedures were completed in a single session, mean number the sessions for cases undergoing RIRS was higher than PCNL group (1.220.05). Mean duration of the procedure was slightly longer in RIRS group when compared with PNL group. Regarding the mean degree of hydronephrosis in both groups, there was a statistically significant difference in favour of cases undergoing PNL and this could be best explained by the preference of the surgeons for PNL in kidneys with relatively higher degree of dilatation. Last but not least regarding the complications as evaluated on the basis of modified Clavien scoring system although they were similar in both groups; evaluation of mean fluoroscopy time as well as mean $\mathrm{Hb}$ drop rate in both groups clearly revealed them to be higher in cases undergoing PNL procedure.

In the light of our findings and the reported literature data as well, we may say that both PNL and RIRS procedures are equally effective treatment alternatives in the management of renal stones in patients with HSK. Although majority of the patients were stone free after a single session of PNL, taking the more invasive nature of this approach with certain complications (bleeding, extravasation), we believe that RIRS may prove itself a valuable alternative with comparable stone free rates despite possible repeated sessions. Morover, higher risk of radiation exposure as demonstrated in our cases may be another disadvantage of PNL. Depending on the experience of the surgeon and the availability of these systems, best management plan could be made in an individualized basis by considering the patient as well as stone related factors.

The major limitation of the current study may be the retrospective nature of our trial and the lack of randomization. Additionally management of the cases by 7 different surgeons with variable level of experience could constitute another drawback. However, taking the limited number of studies in small series of cases available in the literature, we believe that as the first trial on this subject comparing RIRS vs PCNL in renal stone cases with HSK into account, our findings will be contributive enough to the existing literature.

\section{Conclusions}

Management of renal stones could be challenging depending on the anatomy as well as stone related fac- tors. Of the available minimally invasive treatment alternatives, in the light of the lower stone free rates with SWL reported in the published data; both PNL and RIRS could be safe and effective alternatives for renal stone removal in patients with HSK. However, we belive that a proper plan could be instituted by considering the experience of the surgeon and availability of the technical equipment. Further studies with larger series of cases focusing on the comparative evaluation of both procedures are certainly needed.

\section{References}

1. Bauer S. Anomalies of the upper urinary tract. In: Walsh PC, Retic AB, Vaughan ED, et al. ed. Campbell's Urology, 8th ed. Philadelphia: Elsevier Saunders. 2002.

2. Weizer AZ, Silverstein AD, Auge BK, et al. Determining the incidence of horseshoe kidney from radiographic data at a single institution. J Urol. 2003; 170:1722-6.

3. Raj GV, Auge BK, Weizer AZ, et al. Percutaneous management of calculi within horseshoe kidneys. J Urol. 2003; 170:48-51.

4. Yohannes P, Smith AD. The endourological management of complications associated with horseshoe kidney. J Urol. 2002; 168:5-8.

5. Demirkesen O, Yaycioglu O, Onal B, et al. Extracorporeal shockwave lithotripsy for stones in abnormal urinary tracts: analysis of results and comparison with normal urinary tracts. J Endourol. $2001 ; 15: 681-5$.

6. Kirkali Z, Esen AA, Mungan MU. Effectiveness of extracorporeal shockwave lithotripsy in the management of stone-bearing horseshoe kidneys. J Endourol. 1996; 10:13-5.

7. Sheir KZ, Madbouly K, Elsobky E, et al. Extracorporeal shock wave lithotripsy in anomalous kidneys: 11-year experience with two second-generation lithotripters. Urology. 2003; 62:10-5.

8. Viola D, Anagnostou T, Thompson TJ, et al. Sixteen years of experience with stone management in horseshoe kidneys. Urol Int. 2007; 78:214-8.

9. Miller NL, Matlaga BR, Handa SE, et al. The presence of horseshoe kidney does not affect the outcome of percutaneous nephrolithotomy. J Endourol. 2008; 22:1219-25.

10. Symons SJ, Ramachandran A, Kurien A, et al. Urolithiasis in the horseshoe kidney: a single-centre experience. BJU Int. 2008; 102:1676-80.

11. Ishii H, Rai B, Traxer O, et al. Outcome of ureteroscopy for stone disease in patients with horseshoe kidney: Review of world literature. Urology Annals. 2015; 7:470-4.

12. Dindo D, Demartines N, Clavien PA. Classification of surgical complications: a new proposal with evaluation in a cohort of 6336 patients and results of a survey. Ann Surg. 2004; 240:205-13.

13. Stening SG, Bourne S. Supracostal percutaneous nephrolithotomy for upper pole caliceal calculi. J Endourol. 1998; 12:359-62.

14. Skolarikos A, Binbay M, Bisas A, et al. Percutaneous nephrolithotomy in horseshoe kidneys: factors affecting stone-free rate. J Urol. 2011; 186:1894-8.

15. Turk C, Knoll T, Petrik A, et al. Guidelines on urolithiasis. European Urological Association Web site. http://www.uroweb. org/gls/pdf/22\%20Urolithiasis_LR.pdf. Updated 2014.

16. de la Rosette JJ, Opondo D, Daels FPJ, et al. Categorisation of 
complications and validation of the Clavien score for percutaneous nephrolithotomy. Eur Urol. 2012; 62:246-55.

17. Al-Otaibi K, Hosking DH. Percutaneous stone removal in horseshoe kidneys. J Urol. 1999; 162:674-7.

18. Etemadian M, Maghsoudi R, Abdollahpour V, et al. Percutaneous nephrolithotomy in horseshoe kidney: our 5-year experience. Urology Journal. 2013; 10:856-60.

19. El Ghoneimy MN, Kodera AS, Emran AM, et al. Percutaneous nephrolithotomy in horseshoe kidneys: is rigid nephroscopy sufficient tool for complete clearance? A case series study. BMC Urol. 2009; 9:17.

20. Grasso M, Ficazzola M. Retrograde ureteropyeloscopy for lower pole caliceal calculi. J Urol. 1999; 162:1904-8.
21. Weizer AZ, Springhart WP, Ekeruo WO, et al. Ureteroscopic management of renal calculi in anomalous kidneys. Urology. 2005; 65:265-9.

22. Molimard B, Al-Qahtani S, Lakmichi A, et al. Flexible ureterorenoscopy with holmium laser in horseshoe kidneys. Urology. 2010; 76:1334-7.

23. Atis G, Resorlu B, Gurbuz C, et al. Retrograde intrarenal surgery in patients with horseshoe kidneys. Urolithiasis. 2013; 41:79-83.

24. Gokce IM, Tokath Z, Suer E, et al. Comparison of shock wave lithotripsy (SWL) and retrograde intrarenal surgery (RIRS) for treatment of stone disease in horseshoe kidney patients. IBJU. 2016; 42:96-100.

\section{Correspondence}

Bilal Eryildirim, MD Associate Professor

bilaleryildirim@yahoo.com

Tuncer $M, M D$

Health Sciences University, Dr. Lütfi Kirdar Training and Research Hospital,

Urology Clinic, Tecerdagi Cad. Yakutlar Sitesi G/l1 - Kartal/Istanbul, Turkey

Kucuk EV, MD

Tahra A, MD

Health Sciences University, Umraniye Training and Research Hospital,

Urology Clinic, İstanbul, Turkey

Atis $G, M D$

Turan T, MD

Istanbul Medeniyet University, Goztepe Training and Research Hospital,

Urology Clinic, Istanbul, Turkey

Ozturk M, MD

Koca O, MD

Health Sciences University, Haydarpasa Numune Training and Research Hospital, Urology Clinic, Istanbul, Turkey

Senkul T, MD

Ates F, MD

Yilmaz $O, M D$

Health Sciences University, Sultan Abdulhamid Han Training and Research Hospital, Urology Clinic, Istanbul, Turkey

Gurbuz C, MD

Medistate Hospital, Urology Department, Istanbul, Turkey

Sarica K, MD

Kafkas University, Faculty of Medicine, Urology Department

Kafkas, Turkey 